\title{
Evaluación del Cambio en los Participantes del Programa Intergeneracional de Educación Primaria "Compartir la Infancia"
}

\section{Evaluation of Change in the Participants of the Primary Education Intergenerational Programme "Sharing Childhood"}

\author{
Carmen Orte, Marga Vives, Joan Amer, Victoria Quesada* \\ Universitat de les Illes Balears, España
}

\begin{abstract}
DESCRIPTORES:
Educación primaria

Programa

intergeneracional

Relaciones

intergeneracionales

Actitudes

Diseño cuasi-

experimental

\section{RESUMEN:}

Las relaciones intergeneracionales implican una interacción, cooperación e intercambio entre personas de diferentes edades. En una sociedad como la nuestra, cada vez más fraccionada por generaciones, los programas intergeneracionales escolares pretenden reducir esta brecha fomentando lazos positivos a través del contacto entre niños y mayores, reduciendo el edadismo y los prejuicios hacia los mayores. Este artículo pretende analizar si, tras la aplicación de un programa intergeneracional escolar, Compartir la Infancia, se detectan cambios en las actitudes de los estudiantes y los mayores participantes. Para ello, se llevó a cabo un diseño de investigación pre-test post-test con grupo cuasi-experimental y control en el que participaron 274 estudiantes de 12 aulas de quinto y sexto de tres centros de Educación Primaria y 40 personas mayores. Los resultados muestran que el programa intergeneracional influye positivamente en las actitudes de los estudiantes, produciéndose una mejora estadísticamente significativa en las actitudes personales hacia los mayores y en la percepción sobre su sociabilidad y relaciones. Asimismo, tras la participación en el programa se produce una mejora en las actitudes de las personas mayores en cuanto a sus expectativas ante la vejez, así como en sus capacidades. Por otra parte, no se perciben cambios en las actitudes de los mayores hacia los niños.
\end{abstract}

KEYWORDS:
Intergenerational
programs
Intergenerational
relationships
Primary education;
Attitudes
Quasi-experimental
design

\begin{abstract}
:
Intergenerational relationship is understood as the interaction, cooperation and exchange between people of different age groups. It is essential in an ever-growing compartmentalised society. The intergenerational programmes developed in school settings aim to reduce the gap among generations fostering positive ties facilitating contact between students and elders. This paper aims to analyse whether the participation in an intergenerational programme, "Sharing Childhood", affects students and elders' attitudes towards each other and towards ageing. To this end, a pre-test post-test research design with quasi-experimental and control groups was implemented. 274 students, from three primary education schools and 40 elders participated in the study. The results show that the programme seems to have a positive effect on the participating students, resulting in an enhancement in personal attitudes towards elders and their perception of elder's sociability. Likewise, the programme seems to enhance the scores of the elders regarding their ageing expectations and the elder's capacity. However, it does not seem to have an effect on their personal attitudes towards children, among others.
\end{abstract}

CÓMO CITAR:

Orte, C., Vives, M., Amer, J. y Quesada, V. (2021). Evaluación del cambio en los participantes del programa intergeneracional de educación primaria “compartir la infancia”. Revista Iberoamericana de Evaluación Educativa, 14(2), 5-19. https://doi.org/10.15366/riee2021.14.2.001

*Contacto: victoria.quesada@uib.eu

ISSN: 1989-0397

https://revistas.uam.es/riee 


\section{Introducción}

En una sociedad cada vez más compartimentada por generaciones (Kaplan, 2001; Veelken, 2001) en la que se establece un contacto mayoritario con personas nacidas en el mismo periodo temporal, se hace necesario promover el vínculo y el conocimiento intergeneracional, esto es, el contacto con personas de franjas de edades diferentes a la propia, con el fin de mejorar así los lazos sociales y fomentar la comunicación positiva entre sus miembros (Hatton-Yeo, 2015).

Los programas intergeneracionales implican una interacción sistematizada y continuada entre miembros de diferentes grupos de edades para obtener un beneficio mutuo (Newman et al., 1997). Concretamente, los programas intergeneracionales escolares suponen una forma de aprendizaje que tienen como objetivo reunir a las personas en actividades que promuevan una comprensión y respeto entre generaciones, superando posibles actitudes negativas que puedan existir entre ellas (Sánchez et al., 2018), creando relaciones significativas y fomentando su crecimiento emocional y social (Newman y Hatton-Yeo, 2008). Estos programas permiten abrir la escuela a la comunidad en favor de una escuela receptiva a todas las edades, por tanto, siendo más inclusiva (Abarrategui, 2018; Orozco y Moriña, 2020) y pueden fomentar una educación para la ciudadanía global, que pretende, como indican Blasco-Serrano y colaboradores (2019), ayudar a facilitar la comprensión del mundo y del otro para poder comprenderse mejor. La participación activa de personas mayores en un centro escolar, además, permite potenciar la vinculación del centro y del alumnado con el barrio y el sentido de pertenencia. Del mismo modo, se reduce el sentimiento de soledad de los mayores (Murayama et al., 2015) aprovechando su enorme potencial social y fomenta el interés del estudiantado por acercarse a sus abuelos y a otras personas de mayor edad (Lozano Martínez et al., 2018). Al mismo tiempo, el contacto y la interrelación entre personas de diferentes grupos de edad mejora la autoestima de los mayores (Vives et al., 2016) y su bienestar, incrementa la calidad de vida de todos los implicados (Pinazo y Kaplan, 2007), el beneficio mutuo (Moreno Abellán et al., 2018) y aumenta la solidaridad y la percepción hacia otras generaciones (Chung y Kim, 2020).

\section{Revisión de la literatura}

Si bien son numerosos los beneficios que se relacionan con los programas intergeneracionales a nivel internacional, la investigación sobre los cambios en las actitudes de las personas mayores y los niños después de su participación en programas intergeneracionales es un enfoque todavía incipiente y poco desarrollado en el ámbito de la gerontología educativa (Cohen-Mansfield y Jensen, 2017; Cummings et al., 2008; Lynott y Merola, 2007; Osborne y Hall, 2008; Thompson y Weaver, 2016; Whitehouse, 2013; Whitehouse et al., 2000, 2016). También a nivel nacional la evaluación de los cambios producidos tras la participación en un programa intergeneracional se encuentra en un estado emergente, si bien, cabe destacar las investigaciones desarrolladas por Guerra (2014), Pinazo y Montoro (2008) y Sánchez y otros (2018).

Diferentes estudios se centran en analizar las actitudes implícitas de los jóvenes (Babcock et al., 2016; Kogan y Schoenfeld-Tacher, 2018). Kogan y Schoenfeld-Tacher (2018) señalaron la importancia de medir el edadismo presente de forma implícita en las generaciones más jóvenes dada la asociación de éste con los comportamientos con prejuicio hacia las generaciones mayores. De hecho, en las medidas implícitas en un estudio desarrollado por Babcock y colaboradores (2016) los niños mostraron una preferencia clara hacia las personas jóvenes en lugar de hacia las personas mayores, mientras que las medidas explícitas no revelaban esta preferencia.

En la franja de edades entre 6 y 12 años, existen programas intergeneracionales evaluados centrados en una gran diversidad de aspectos. Son ejemplos de ello los programas orientados a la mejora de la lectura acompañados de un senior (Doiron y Lees, 2009; Isaki y Harmon, 2015) o los programas escolares que combaten la brecha digital de los mayores con su acompañamiento a menores en las escuelas en actividades informáticas (Gamilel y Gabay, 2014). Asimismo, en esta franja de edad cabe destacar por su trayectoria el proyecto "The Intergenerational School” (TIS) (Bendezu et al., 2000; Whitehouse, 2013; Whitehouse et al., 2016) en la que personas mayores participan como co-aprendices, produciéndose mejoras a nivel comunitario (George et al., 2011). 
En el ámbito de la población escolar de educación infantil y primaria y la gente mayor, una revisión sistemática internacional sobre el efecto de los programas intergeneracionales en sus actitudes y percepciones muestra resultados positivos tanto en los niños como en los mayores (Gualano et al., 2018). Según los autores, estos resultados positivos son el denominador común de un abanico de investigaciones que utilizan metodologías y técnicas de encuesta y entrevista muy distintas. En concreto afirman que los estudios analizados obtienen mejoras en las percepciones de los niños sobre la gente mayor, y en especial en los niños de educación infantil. Asimismo, en otra revisión sistemática similar (Giraudeau y Bailly, 2019) se perciben mejoras significativas en las actitudes de los niños hacia los mayores, si bien, los beneficios no son sistemáticos en todos los estudios, o bien no se han evaluado adecuadamente. En cuanto a las personas mayores, el aspecto más investigado es el bienestar psicosocial. A pesar de la heterogeneidad de resultados y las diferentes escalas de evaluación utilizadas, la mayoría de estudios analizados en ambas revisiones sistemáticas obtienen resultados positivos (Giraudeau y Bailly, 2019; Gualano et al., 2018).

Igualmente, se señalan una serie de programas intergeneracionales concretos que incorporan la evaluación pre-test y post-test de las actitudes. Fujiwara y colaboradores (2009) mostraron que tras participar en un programa intergeneracional aumentó la frecuencia con la que los mayores se comunicaban con niños. En el caso de la investigación de Lynott y Merola (2007), después de implementar un programa intergeneracional que promueve la interacción social y las actividades artísticas, así como visitas entre residencias de mayores y escuelas se constató que, tras la implementación, los niños percibían de manera más positiva a los mayores. Mejoraba especialmente el considerar a los mayores como personas inteligentes, cálidas, saludables y relajadas. Babcock y colaboradores (2018) detectaron mejoras, pero no cambios estadísticamente significativos después de la aplicación del programa "Bridges Together". Este programa se estructura de manera similar al programa Compartir la Infancia, objeto del presente artículo: seis sesiones con diferentes temáticas y presencia de unos cuatro alumnos sénior (personas mayores) en las clases, permitiendo el trabajo en pequeños grupos. Los aspectos evaluados son relativos al edadismo y el cambio de percepción de los niños tras el programa. Por otro lado, en el programa intergeneracional analizado por Osborne y Hall (2008) se consiguió que los niños tuvieran una percepción más realista de los mayores, especialmente en cuanto a su condición física. Para ello fue crucial, según los autores, el tiempo compartido de calidad en pequeños grupos.

Semejantes resultados se obtuvieron en un estudio centrado en investigar si mejoran las actitudes de los estudiantes después de participar en una actividad artística intergeneracional (Whiteland, 2016). Los análisis estadísticos no detectaron cambios estadísticamente significativos, sin embargo, presentó tendencias de cambio positivas conforme que los estudiantes construyeron nuevos significados en relación a la gente mayor. Por otra parte, en Dunham y Casadonte (2009) se halló que las actitudes específicas de los niños hacia los mayores en las aulas eran los factores predictores más importantes a la hora de pedir ayuda a un mayor. Estos autores apuntan que los programas con voluntarios mayores en las aulas pueden tener un efecto positivo en las actitudes de los niños, incluso cuando el propósito explícito del programa no sea el cambio de actitud. Así, indican que el proceso de trabajar juntos, conocerse de manera más personal y trabajar para un objetivo común puede reducir las actitudes negativas de los niños hacia el envejecimiento (Dunham y Casadonte, 2009).

El objetivo del presente estudio es, precisamente, ahondar en esta línea de investigación examinando si tras la participación en un programa intergeneracional desarrollado con alumnado de quinto y sexto de Educación primaria y personas mayores se producen cambios, por una parte, en las actitudes del alumnado hacia los mayores, y por otra, en las actitudes de los mayores hacia los niños, así como en sus percepciones hacia la vejez.

\section{Contexto: Programa intergeneracional compartir la infancia}

El programa Compartir la Infancia (Sharing Childhood-SACHI2) está alineado con los objetivos europeos sobre el aprendizaje a lo largo de toda la vida; en concreto, la necesidad de incrementar la participación de las personas mayores en diferentes contextos de aprendizaje (Education and Training, 2020). Concretamente, trabaja sobre los siguientes objetivos estratégicos: 
- Hacer realidad el aprendizaje permanente (adquisición de nuevas habilidades en diferentes ámbitos en las personas mayores).

- Mejorar la calidad y la eficiencia de la educación, promoviendo proyectos innovadores fácilmente adaptables a las realidades sociales del entorno y favoreciendo su inclusión en el currículum escolar.

- Promover la equidad, la cohesión social y la ciudadanía activa; permitiendo a las personas mayores ser agentes activos en su comunidad, favoreciendo la cohesión social entre generaciones.

- Aumentar la creatividad y la innovación en todos los niveles educativos; en concreto, en la segunda etapa de primaria (9-12 años).

Se parte de la concepción de que el intercambio y establecimiento de relaciones positivas entre diferentes generaciones, en este caso entre niños y personas mayores, genera beneficios importantes en los participantes, mejora el aprendizaje y la motivación del alumnado (Orte et al., 2018; Vives et al., 2016) así como el bienestar físico, psicológico y social en los mayores (Gutiérrez Sánchez y Hernández Torrano, 2013).

El programa intergeneracional Compartir la infancia se desarrolla en el centro educativo. Tiene una duración de entre 3 y 4 meses y requiere de la participación en el aula de una serie de agentes de dentro y fuera del centro. Para su desarrollo se hace necesaria la implicación del profesor-tutor del aula, del profesorado de lengua extranjera, del alumnado y de personas mayores voluntarias, a las que se denomina seniors. Las personas mayores pueden provenir de diferentes lugares, preferentemente del mismo barrio (a través de centros de personas mayores, centros de día, etc.) o del propio centro (abuelos de otros grupos, por ejemplo), ello permite la sostenibilidad y difusión del proyecto en su comunidad.

El programa consta de ocho sesiones de trabajo conjunto entre estudiantes y personas mayores, que se desarrollan en semanas alternas. Si bien el alumnado asiste a 8 sesiones, los seniors asisten semanalmente al centro escolar, participando en 16 sesiones, alternando una semana para la preparación de la actuación con los docentes (coordinación) y la siguiente para el trabajo conjunto con el alumnado (implementación). Destacar que tanto las sesiones de implementación como las de coordinación se realizan en horario escolar.

Las reuniones de coordinación, en la que participan los senior, docente especialista y tutor, permiten realizar un seguimiento y evaluación de la sesión anterior, así como una planificación de la siguiente, pudiendo colaborar todas las partes aportando ideas. Las sesiones de implementación tienen una duración de una hora y media aproximadamente y se desarrollan en el aula. Durante estas sesiones, el aula se organiza en grupos de trabajo colaborativo, formados por seis estudiantes y una persona voluntaria mayor, con la guía del docente-tutor y/o del especialista de inglés. Es importante resaltar que la elección de la estrategia del aprendizaje colaborativo no es casual, pues se pretende favorecer una mayor interacción. Asimismo, se propone que los grupos de trabajo colaborativos se mantengan estables durante toda la implementación del programa con el fin de favorecer la formación de un vínculo mayor entre los estudiantes y la persona senior.

De las ocho sesiones de trabajo conjunto, la primera es de presentación y la última de clausura. Durante las 6 sesiones intermedias, se trabajan temas específicamente seleccionados para facilitar la relación y el conocimiento mutuo entre los participantes, permitiendo compartir su infancia. Así, se trabajan temas que estudiantes y personas mayores tienen en común, estructurados en: 1) La Familia, 2) Ídolos y referentes, 3) La escuela de hoy y de ayer, 4) Juegos populares, 5) El barrio y 6) Fiestas populares. A través de estos temas, y de las actividades diseñadas para tal fin, se permite la comunicación y el intercambio de ideas entre el alumnado y los seniors, así como la comparación entre las situaciones vividas favoreciendo la discusión y el reconocimiento de puntos comunes y divergentes. Indicar también que en todas las sesiones se incorporan actividades específicas de escritura, así como vocabulario en lengua extranjera y el uso de tecnología (mapas en línea, búsquedas en internet, etc.).

Cabe mencionar que la organización es la base para la sostenibilidad del programa intergeneracional en cada centro educativo. La implementación del programa no supone gasto económico y se permite su adap- 
tación en función de las características y necesidades del centro educativo donde se realice. Para ello, la guía de implementación del programa recomienda, entre otras orientaciones, desarrollarlo desde la perspectiva del trabajo en red (fomentando que las instituciones comunitarias se involucren), aprovechar el conocimiento adquirido durante los meses de trabajo para adaptarlo e implementarlo en otros grupos dentro del centro escolar o hacer difusión de la experiencia.

\section{Método}

\section{Enfoque metodológico}

Con el fin de dar respuesta a los objetivos planteados se ha llevado a cabo un estudio cuasi-experimental siguiendo un diseño con grupo cuasi-experimental y control con medidas pre-test y post-test. El tratamiento recibido por el grupo cuasi-experimental consistió en el programa intergeneracional Compartir la infancia. El grupo control no recibió ningún tipo de tratamiento.

\section{Muestra y muestreo}

Para la selección de la muestra se establecieron los siguientes criterios:

- Estudiantes de entre 9 y 12 años de escuelas de educación primaria, preferentemente públicas, de distintos barrios de la ciudad de Palma.

- Personas voluntarias a partir de 55 años, activas, preferentemente procedentes del barrio del centro escolar.

La muestra productora de datos finalmente está formada por 275 estudiantes de cuarto y quinto de primaria de 12 aulas de tres centros educativos de la ciudad de Palma y de 40 seniors. Se ofreció la oportunidad de participar a diversos centros escolares, y finalmente se eligieron tres centros de tres barrios de Palma con características diferenciadas. Los seniors, por otra parte, eran personas voluntarias y dispuestas a participar en el programa. Cabe destacar que el grupo de seniors era heterogéneo entre sí en cuanto a edades se refiere, comprendiendo desde los 55 hasta los 84 años.

Para la asignación de los seniors a las aulas (4-5 por aula), se decidió buscar un equilibrio en cuanto a la edad, la procedencia, de forma que al menos la mitad de los seniors del aula fueran del propio barrio para favorecer la vinculación con el entorno, el sentido de pertenencia y la sostenibilidad del programa. Y el sexo, si bien hubo un número mayor de mujeres voluntarias, se asignó uno o dos hombres a cada grupo.

En el Cuadro 1 se puede apreciar la distribución de la muestra en los grupos cuasi-experimental y control en cada uno de los centros y aulas. El grupo cuasi-experimental está comprendido por 145 estudiantes y 27 seniors repartidos en 6 aulas y el grupo control por 130 estudiantes de otras 6 aulas y 18 seniors.

Cuadro 1

Distribución de la muestra de cada grupo por centro y aula

\begin{tabular}{|c|c|c|c|c|c|}
\hline & & Centro 1 & Centro 2 & Centro 3 & Total \\
\hline \multirow{4}{*}{$\begin{array}{l}\text { Grupo } \\
\text { cuasi-experimental }\end{array}$} & $\mathrm{N}^{0}$ aulas & 2 & 2 & 2 & 6 \\
\hline & Curso & Cuarto & Quinto & Quinto & - \\
\hline & $\mathrm{N}^{0}$ estudiantes & 50 & 52 & 43 & 145 \\
\hline & $\mathrm{N}^{0}$ senior & 9 & 9 & 9 & 27 \\
\hline \multirow{4}{*}{ Grupo control } & $\mathrm{N}^{0}$ aulas & 2 & 2 & 2 & 6 \\
\hline & Curso & Quinto & Cuarto & Cuarto & - \\
\hline & No estudiantes & 45 & 47 & 38 & 130 \\
\hline & $\mathrm{N}^{\circ}$ de senior & 5 & 4 & 4 & 13 \\
\hline
\end{tabular}


Como se puede apreciar los grupos cuasi-experimental y control proceden de los mismos centros. No obstante, y aunque los tres centros cuentan con dos líneas por curso, señalar que, por cuestiones éticas se decidió aplicar el programa en las dos líneas del curso del mismo centro, de modo que, en los centros en los que se aplicó el programa en cuarto de primaria, los estudiantes de quinto ejercían de grupo control y en los que se aplicó en quinto de primaria, los estudiantes de cuarto actuaban de grupo control.

\section{Instrumentos de obtención de información}

Para analizar el cambio producido en las actitudes se han utilizado dos cuestionarios, ambos son adaptaciones del cuestionario de actitudes hacia las personas mayores, elaborado y validado por Montoro (1998) para población universitaria. Dicho instrumento fue modificado y adaptado a la población diana: alumnado de cuarto y quinto de Educación Primaria y personas mayores de 55 años. Concretamente se realizaron dos versiones del instrumento.

Para la adaptación del cuestionario a las edades del alumnado de primaria se redujeron considerablemente el número de ítems, del mismo modo, se modificó la redacción para hacerla más comprensible para niños de estas edades. Una vez modificado el instrumento se realizó una prueba piloto con niños y niñas de cuarto de educación primaria de un centro de educación público de Palma, diferente a los que participaron posteriormente como grupo experimental y control. A partir de esta prueba se modificó la redacción de diversos ítems para hacerlo más comprensible. Del mismo modo, en la adaptación del cuestionario para personas mayores, se incluyó una nueva dimensión (actitudes hacia los niños) y realizó una prueba piloto, modificándose la redacción de dos ítems para hacerlos más claros. Resaltar que en ambos cuestionarios el rango de respuesta de los ítems es del 1 al 4, siendo 1 "no estoy nada de acuerdo" y 4 "estoy totalmente de acuerdo".

En el cuestionario para seniors se presentan 44 afirmaciones para conocer sus actitudes en relación al envejecimiento y sus actitudes respecto a los niños, englobadas en siete dimensiones: 1) Actitud ante el proceso de envejecimiento, 2) Nivel de discriminación y prejuicio presente en la sociedad contra los mayores, 3) Capacidad de los mayores, 4) Nivel de sociabilidad de los mayores, 5) Actitudes personales hacia los niños, 6) Expectativas ante la vejez, y 7) Problemas de la vejez.

El cuestionario para estudiantes presenta 26 afirmaciones en relación a sus actitudes hacia las personas mayores. Al modificarse de forma sustancial el cuestionario original para su adaptación a niños y niñas a partir de 9 años, se realizó un análisis factorial para valorar la nueva estructura de las 25 afirmaciones del cuestionario que analiza las percepciones y actitudes de 275 alumnos de cuarto y quinto de primaria en relación a las personas mayores y al envejecimiento. La puntuación Kaiser-Meyer-Olkin (KMO) obtenida en su globalidad fue de 0,7 puntos. El test de esfericidad de Bartlett fue estadísticamente significativo ( $p$ $<0,0001$ ), indicando que los datos eran susceptibles de ser factorizados. Tras el análisis factorial, una vez eliminados los cinco ítems que no presentaban una correlación positiva superior a 0,4 se obtuvieron 6 componentes con un eigenvalue superior a uno, estos seis componentes explican un 51,17\% de la varianza. Se utilizó la rotación ortogonal Varimax con normalización de Kaiser para facilitar la interpretación, la rotación convergió en ocho iteracciones. Los 6 componentes obtenidos se han denominado de la siguiente forma:

- Dimensión 1. Actitudes personales hacia los mayores.

- Dimensión 2. Capacidad de concentración y atención al detalle de los mayores.

- Dimensión 3. Actitudes personales hacia los mayores II.

- Dimensión 4. Capacidad de los mayores de realizar actividades y tareas.

- Dimensión 5. Sociabilidad y relaciones de los mayores.

- Dimensión 6. Percepción personal sobre su futuro como persona mayor.

\section{Trabajo de campo}

El programa intergeneracional Compartir la Infancia se desarrolló entre el primer y segundo trimestre del curso. En la primera sesión del programa intergeneracional se administraron los cuestionarios 
pre-test, uno al alumnado de educación primaria y el otro a los seniors, de forma presencial. Se aplicó en el aula y bajo la supervisión del maestro/a de educación primaria y de dos miembros del equipo de investigación. Igualmente, en la última sesión se administraron de nuevo ambos cuestionarios, en las mismas condiciones ambientales. En el mismo período de tiempo y siguiendo las mismas condiciones (en el aula y con la supervisión del maestro y de dos miembros del equipo de investigación) contestaron los cuestionarios pre-test y post-test los participantes del grupo control.

\section{Análisis de datos}

Los datos obtenidos en este estudio han sido analizados utilizando el programa SPSS 25 . Se han calculado estadísticos descriptivos con medidas de tendencia central y dispersión. Asimismo, siguiendo las indicaciones de Connolly (2007) para el análisis de las puntuaciones en diseños cuasi-experimentales con medidas pre-test y post-test de dos grupos (experimental y control), se ha utilizado la regresión lineal múltiple con el fin de analizar si se producen cambios estadísticamente significativos en las actitudes del alumnado de primaria y en los seniors tras participar en el programa intergeneracional, tomándose como variable dependiente la puntuación post-test y como variables independientes la puntuación pre-test y el grupo de procedencia (cuasi-experimental o de control) con el fin de controlar las posibles diferencias ya existentes entre las puntuaciones pre-test de ambos grupos.

\section{Resultados}

Se presentan, en un primer momento, los cambios en las actitudes del alumnado detectadas tras la aplicación del programa Compartir la infancia, y, a continuación, de los y las seniors.

\subsection{Cambios en la actitud hacia las personas mayores del alumnado de educación primaria}

En el Cuadro 2 se presentan la media y desviación típica obtenidas por cada componente (a los que denominamos dimensión) y grupo en las medidas pre-test y post-test una vez considerada la carga factorial de cada uno de los ítems que comprenden la dimensión.

Cuadro 2

Media y desviación típica de las dimensiones del alumnado por grupo y momento

\begin{tabular}{lcccccccc}
\hline & \multicolumn{3}{c}{ Grupo cuasi-experimental } & & \multicolumn{2}{c}{ Grupo control } \\
\cline { 2 - 9 } & \multicolumn{2}{c}{ Pre-test } & Post-test & Pre-test & & Post-test \\
\cline { 2 - 9 } & M & DT & M & DT & M & DT & M & DT \\
\hline $\begin{array}{l}\text { 1. Actitudes personales hacia las } \\
\text { personas mayores }\end{array}$ & 2,10 & 0,37 & 2,12 & 0,40 & 1,98 & 0,46 & 1,92 & 0,44 \\
$\begin{array}{l}\text { 2. Capacidad de concentración y } \\
\text { atención al detalle de los mayores }\end{array}$ & 2,01 & 0,41 & 2,05 & 0,41 & 1,95 & 0,44 & 1,97 & 0,41 \\
$\begin{array}{l}\text { 3. Actitudes personales hacia las } \\
\text { personas mayores II }\end{array}$ & 1,96 & 0,59 & 2,11 & 0,54 & 1,88 & 0,61 & 1,94 & 0,57 \\
$\begin{array}{l}\text { 4. Capacidad de los mayores de } \\
\text { realizar actividades y tareas }\end{array}$ & 1,38 & 0,35 & 1,48 & 0,35 & 1,37 & 0,36 & 1,49 & 0,35 \\
$\begin{array}{l}\text { 5. Sociabilidad y relaciones de los } \\
\text { mayores }\end{array}$ & 1,62 & 0,35 & 1,71 & 0,32 & 1,56 & 0,39 & 1,55 & 0,37 \\
$\begin{array}{l}\text { 6. Percepción personal sobre futuro } \\
\text { como persona mayor }\end{array}$ & 2,15 & 0,41 & 2,16 & 0,36 & 2,15 & 0,39 & 2,15 & 0,40 \\
\hline
\end{tabular}

Nota. Se ha calculado la media ponderada en función de la carga de cada ítem al componente (dimensión). 
En una primera aproximación a los datos (Cuadro 2) parece existir una tendencia positiva en las puntuaciones obtenidas por el grupo cuasi-experimental en las seis dimensiones analizadas. En el grupo control, del mismo modo, parece existir una tendencia positiva en tres dimensiones y negativa en dos de ellas. Los resultados de la regresión lineal múltiple (Cuadro 3) muestran que tras la implementación del programa intergeneracional Compartir la Infancia se produce una mejoría estadísticamente significativa en las actitudes del alumnado participante (grupo cuasi-experimental) en tres dimensiones: 1- Actitudes personales hacia los mayores, 3- Actitudes personales hacia los mayores II y 5 - Percepción sobre la sociabilidad y relaciones de los mayores. No obstante, no existen diferencias estadísticamente significativas entre los grupos cuasi-experimental y control en las otras tres: 2- Percepción sobre la capacidad de concentración y atención al detalle de los mayores, 4- Percepción sobre la capacidad de realizar actividades y tareas de los mayores y 6 - Percepción personal hacia el futuro como adulto (perspectivas de su futuro como personas mayores). Pues, tal y como se aprecia en el Cuadro 3 las puntuaciones post-test obtenidas no se ven afectadas por la variable grupo (cuasi-experimental y control) una vez consideradas las puntuaciones pre-test. Por último, indicar que el modelo no se vio afectado por la variable género.

Cuadro 3

Regresión lineal múltiple del análisis de las puntuaciones en los niños y niñas de Educación Primaria

\begin{tabular}{|c|c|c|c|c|c|c|c|}
\hline & & \multicolumn{2}{|c|}{ Coef. no estand. } & \multirow{2}{*}{$\begin{array}{c}\begin{array}{c}\text { Coef. } \\
\text { estand. }\end{array} \\
\text { B }\end{array}$} & \multirow{2}{*}{$t$} & \multirow{2}{*}{ sig. } & \multirow{2}{*}{$\begin{array}{l}\text { Ajuste del } \\
\text { modelo }\end{array}$} \\
\hline & & B & $\mathrm{DE}$ & & & & \\
\hline \multirow{3}{*}{$\begin{array}{l}\text { 1. Actitudes personales hacia los } \\
\text { mayores }\end{array}$} & Constante & 1,263 & 0,140 & & 9,035 & 0,00 & \multirow{3}{*}{$\begin{array}{c}\mathrm{p}<0,01 \\
\mathrm{~F}=47,956 \\
\mathrm{gl}=2,272\end{array}$} \\
\hline & Momento & 0,474 & 0,054 & 0,459 & 8,711 & 0,00 & \\
\hline & Grupo & $-0,142$ & 0,045 & $-0,165$ & $-3,135$ & $0,002^{*}$ & \\
\hline \multirow{3}{*}{$\begin{array}{l}\text { 2. Capacidad de concentración y } \\
\text { atención al detalle de los mayores }\end{array}$} & Constante & 1,336 & 0,132 & & 10,121 & 0,00 & \multirow{3}{*}{$\begin{array}{c}\mathrm{p}<0,01 \\
\mathrm{~F}=26,699 \\
\mathrm{gl}=2,272\end{array}$} \\
\hline & Momento & 0,382 & 0,054 & 0,395 & 7,100 & 0,00 & \\
\hline & Grupo & $-0,055$ & 0,046 & $-0,067$ & $-1,204$ & 0,230 & \\
\hline \multirow{3}{*}{$\begin{array}{l}\text { 3. Actitudes personales hacia los } \\
\text { mayores }\end{array}$} & Constante & 1,993 & 0,152 & & 13,141 & 0,00 & \multirow{3}{*}{$\begin{array}{c}\mathrm{p}<0,01 \\
\mathrm{~F}=6,873 \\
\mathrm{gl}=2,272\end{array}$} \\
\hline & Momento & 0,144 & 0,055 & 0,156 & 2,624 & 0,009 & \\
\hline & Grupo & $-0,161$ & 0,66 & $-0,145$ & $-2,440$ & $0,015^{*}$ & \\
\hline \multirow{3}{*}{$\begin{array}{l}\text { 4. Capacidad de los mayores de } \\
\text { realizar actividades }\end{array}$} & Constante & 1,058 & 0,100 & & 10,536 & 0,00 & \multirow{3}{*}{$\begin{array}{c}\mathrm{p}<0,01 \\
\mathrm{~F}=14,405 \\
\mathrm{gl}=2,272\end{array}$} \\
\hline & Momento & 0,302 & 0,056 & 0,310 & 5,367 & 0,00 & \\
\hline & Grupo & 0,007 & 0,04 & 0,010 & 0,177 & 0,86 & \\
\hline \multirow{3}{*}{$\begin{array}{l}\text { 5. Sociabilidad y relaciones de los } \\
\text { mayores }\end{array}$} & Constante & 1,454 & 0,110 & & 13,257 & 0,00 & \multirow{3}{*}{$\begin{array}{c}\mathrm{p}<0,01 \\
\mathrm{~F}=18,556 \\
\mathrm{gl}=2,272\end{array}$} \\
\hline & Momento & 0,248 & 0,054 & 0,262 & 4,591 & 0,00 & \\
\hline & Grupo & $-0,145$ & 0,040 & $-0,207$ & $-3,630$ & $0,00^{*}$ & \\
\hline \multirow{3}{*}{$\begin{array}{l}\text { 6. Percepción personal hacia el } \\
\text { futuro como adulto }\end{array}$} & Constante & 1,681 & 0,138 & & 12,174 & 0,00 & \multirow{3}{*}{$\begin{array}{c}\mathrm{p}<0,01 \\
\mathrm{~F}=8,437 \\
\mathrm{gl}=2,272\end{array}$} \\
\hline & Momento & 0,228 & 0,056 & 0,241 & 4,103 & 0,00 & \\
\hline & Grupo & $-0,009$ & 0,044 & $-0,012$ & $-0,196$ & 0,845 & \\
\hline
\end{tabular}

Nota. ${ }^{*} \mathrm{p}<0,05$. 


\subsection{Cambios en la actitud en relación al envejecimiento y hacia los niños}

En el Cuadro 4 se presentan las puntuaciones medias y desviación típica obtenidas por las personas mayores del grupo cuasi-experimental y control para cada una de las dimensiones analizadas en el pre-test y en el post-test. Tal y como ocurría con el alumnado, se puede observar que existe una tendencia de mejora en todas las del grupo cuasi-experimental. En el grupo control, por otra parte, se aprecia una tendencia positiva en cuatro de ellas y un retroceso en tres.

Cuadro 4

Media y desviación típica en las dimensiones de los y las seniors por grupo y momento

\begin{tabular}{|c|c|c|c|c|c|c|c|c|}
\hline & \multicolumn{4}{|c|}{ Grupo cuasi-experimental } & \multicolumn{4}{|c|}{ Grupo control } \\
\hline & \multicolumn{2}{|c|}{ Pre-test } & \multicolumn{2}{|c|}{ Post-test } & \multicolumn{2}{|c|}{ Pre-test } & \multicolumn{2}{|c|}{ Post-test } \\
\hline & M & DT & M & DT & M & DT & M & DT \\
\hline $\begin{array}{l}\text { 1. Actitud ante el proceso de } \\
\text { envejecimiento }\end{array}$ & 3,00 & 0,41 & 3,15 & 0,49 & 2,64 & 0,46 & 2,76 & 0,56 \\
\hline $\begin{array}{l}\text { 2. Nivel de discriminación y prejuicio } \\
\text { presente en la sociedad contra los } \\
\text { mayores }\end{array}$ & 2,78 & 0,45 & 2,82 & 0,59 & 2,77 & 0,49 & 2,62 & 0,55 \\
\hline 3. Capacidad de los mayores. & 2,53 & 0,60 & 2,75 & 0,58 & 2,48 & 0,53 & 2,49 & 0,56 \\
\hline $\begin{array}{l}\text { 4. Nivel de sociabilidad de los } \\
\text { mayores }\end{array}$ & 2,77 & 0,50 & 3,01 & 0,35 & 2,97 & 0,41 & 2,93 & 0,44 \\
\hline $\begin{array}{l}\text { 5. Actitudes personales hacia los } \\
\text { niños }\end{array}$ & 3,62 & 0,38 & 3,65 & 0,53 & 3,36 & 0,49 & 3,46 & 0,50 \\
\hline 6. Expectativas ante la vejez & 3,02 & 0,42 & 3,17 & 0,42 & 2,65 & 0,52 & 2,61 & 0,64 \\
\hline 7. Problemas de la vejez & 2,75 & 0,67 & 2,70 & 0,56 & 2,54 & 0,50 & 2,58 & 0,55 \\
\hline
\end{tabular}

Con el fin de conocer si se producen cambios estadísticamente significativos en las actitudes de los seniors participantes tras la participación en el programa intergeneracional, y para neutralizar las diferencias entre las puntuaciones pre-test obtenidas, se calculó la regresión lineal múltiple (Cuadro 5). 
Cuadro 5

Regresión lineal múltiple del análisis de las puntuaciones en los y las seniors

\begin{tabular}{|c|c|c|c|c|c|c|c|}
\hline & & \multicolumn{2}{|c|}{ Coef. no estand. } & \multirow{2}{*}{$\begin{array}{l}\begin{array}{l}\text { Coef. } \\
\text { estand. }\end{array} \\
\text { B }\end{array}$} & \multirow{2}{*}{$t$} & \multirow{2}{*}{ sig. } & \multirow{2}{*}{$\begin{array}{c}\text { Ajuste del } \\
\text { modelo }\end{array}$} \\
\hline & & $\mathrm{B}$ & $\mathrm{DE}$ & & & & \\
\hline \multirow{3}{*}{$\begin{array}{l}\text { 1. Actitud ante el proceso de envejeci- } \\
\text { miento }\end{array}$} & Constante & 1,034 & 0,492 & & 2,099 & 0,041 & \multirow{3}{*}{$\begin{array}{l}\mathrm{p}<0,01 \\
\mathrm{~F}=20,74 \\
\mathrm{gl}=2,49\end{array}$} \\
\hline & Momento & 0,748 & 0,136 & 0,625 & 5,485 & 0,000 & \\
\hline & Grupo & $-0,124$ & 0,126 & $-0,113$ & $-0,987$ & 0,328 & \\
\hline \multirow{3}{*}{$\begin{array}{l}\text { 2. Nivel de discriminación y prejuicio } \\
\text { presente en la sociedad contra los } \\
\text { mayores }\end{array}$} & Constante & 0,675 & 0,392 & & 1,721 & 0,092 & \multirow{3}{*}{$\begin{array}{l}\mathrm{p}<0,01 \\
\mathrm{~F}=23,93 \\
\mathrm{gl}=2,49\end{array}$} \\
\hline & Momento & 0,837 & 0,125 & 0,682 & 6,708 & 0,000 & \\
\hline & Grupo & $-0,187$ & 0,116 & $-0,164$ & $-1,615$ & 0,113 & \\
\hline \multirow{3}{*}{ 3. Capacidad de los mayores } & Constante & 0,989 & 0,285 & & 3,474 & 0,001 & \multirow{3}{*}{$\begin{array}{l}\mathrm{p}<0,01 \\
\mathrm{~F}=39,32 \\
\mathrm{gl}=2,49\end{array}$} \\
\hline & Momento & 0,777 & 0,091 & 0,754 & 8,504 & 0,000 & \\
\hline & Grupo & $-0,212$ & 0,102 & $-0,185$ & $-2,083$ & $0,042^{*}$ & \\
\hline \multirow{3}{*}{ 4. Nivel de sociabilidad de los mayores } & Constante & 2,466 & 0,343 & & 7,194 & 0,000 & \multirow{3}{*}{$\begin{array}{l}\mathrm{p}<0,01 \\
\mathrm{~F}=2,326 \\
\mathrm{gl}=2,49\end{array}$} \\
\hline & Momento & 0,240 & 0,117 & 0,286 & 2,044 & 0,046 & \\
\hline & Grupo & $-0,122$ & 0,109 & $-0,158$ & $-1,125$ & 0,266 & \\
\hline \multirow{3}{*}{ 5. Actitudes personales hacia los niños } & Constante & 1,301 & 0,569 & & 2,286 & 0,027 & \multirow{3}{*}{$\begin{array}{c}\mathrm{p}<0,01 \\
\mathrm{~F}=12,325 \\
\mathrm{gl}=2,49\end{array}$} \\
\hline & Momento & 0,654 & 0,139 & 0,573 & 4,712 & 0,000 & \\
\hline & Grupo & $-0,020$ & 0,124 & $-0,020$ & $-0,163$ & 0,871 & \\
\hline \multirow{3}{*}{ 6. Expectativas ante la vejez } & Constante & 1,203 & 0,458 & & 2,628 & 0,011 & \multirow{3}{*}{$\begin{array}{c}\mathrm{p}<0,01 \\
\mathrm{~F}=29,734 \\
\mathrm{gl}=2,49\end{array}$} \\
\hline & Momento & 0,746 & 0,124 & 0,620 & 6,001 & 0,000 & \\
\hline & Grupo & $-0,284$ & 0,124 & $-0,237$ & $-2,293$ & $0,026^{*}$ & \\
\hline \multirow{3}{*}{ 7. Problemas de la vejez } & Constante & 1,022 & 0,353 & & 2,892 & 0,006 & \multirow{3}{*}{$\begin{array}{c}\mathrm{p}<0,01 \\
\mathrm{~F}=18,244 \\
\mathrm{gl}=2,49\end{array}$} \\
\hline & Momento & 0,605 & 0,102 & 0,655 & 5,958 & 0,000 & \\
\hline & Grupo & 0,009 & 0,120 & 0,009 & 0,079 & 0,938 & \\
\hline
\end{tabular}

Nota. ${ }^{*} \mathrm{p}<0,05$

Los resultados muestran que tras la participación en el programa intergeneracional Compartir la Infancia se ha producido un cambio estadísticamente significativo en las actitudes de los seniors participantes en el programa (grupo cuasi-experimental) en dos de las siete dimensiones analizadas, concretamente en 3 - Actitudes en relación a la capacidad de los mayores y 6 - Expectativas ante la vejez.

Del lado contrario, tras la participación no se han detectado cambios estadísticamente significativos en las puntuaciones obtenidas en el grupo de seniors participantes en 5- Actitudes personales hacia los niños. Tampoco se aprecian cambios estadísticamente significativos una vez controladas las puntuaciones pre-test entre los grupos cuasi-experimental y control en las cuatro dimensiones restantes: 1-Actitud ante el proceso de envejecimiento, 2-Nivel de discriminación y prejuicio presente en la sociedad contra los mayores, 4-Nivel de sociabilidad de los mayores y 7-Problemas de la vejez. 


\section{Discusión y conclusiones}

El objetivo del estudio es doble: conocer si tras la participación en un programa intergeneracional escolar se producen cambios estadísticamente significativos en las actitudes del alumnado de educación primaria hacia las personas mayores y, por otra, analizar si se producen cambios en la actitud de las y los seniors participantes en el programa hacia los niños y hacia la vejez. Se partía de la idea de que a través del contacto entre ellos mejorarían las actitudes de unos hacia otros y la disminución de prejuicios. Del mismo modo, se consideró que, a través de la actividad y del contacto de personas mayores con otros mayores, mejorarían sus actitudes hacia el envejecimiento y la vejez.

Como indica Hatton-Yeo (2015), el vínculo intergeneracional puede fomentar la creación de lazos y la comunicación positiva entre personas de diferentes edades. El programa intergeneracional escolar evaluado, Compartir la Infancia, pretende propiciar el contacto entre niños y niñas y personas mayores con el fin de fomentar una comunicación positiva, reducir estereotipos y superar actitudes negativas en relación a otras generaciones y también en relación al envejecimiento.

Tal y como ocurre en programas de características similares (Babcock et al., 2018; Osborne y Hall, 2008), se aprecia una tendencia positiva en la actitud de los niños hacia los mayores tras su participación en el programa Compartir la Infancia. En este caso, además, los resultados muestran un cambio, con una actitud más positiva de los niños hacia las personas mayores, así como una mejora en la percepción de las y los niños sobre la sociabilidad de los seniors. Por contra, el programa no parece haber influido ni positiva ni negativamente en la percepción de los y las niños sobre la capacidad de concentración y atención al detalle de las personas mayores, su capacidad para realizar actividades y tareas, ni tampoco a la percepción personal de los niños hacia su futuro como persona mayor.

En cuanto a las personas mayores, resaltar que no se aprecian cambios en las actitudes de los seniors hacia los niños tras la participación en el programa intergeneracional. Esto puede deberse a que los seniors eran personas que, como se puede apreciar en el análisis descriptivo de los datos, ya tenían unas puntuaciones iniciales (pre-test) elevadas en sus actitudes hacia los niños. Aspecto comprensible éste debido a la necesidad de contar con seniors voluntarios para el buen desarrollo del programa. Asimismo, el programa parece no haber afectado a la actitud de los seniors ante el proceso de envejecimiento, su percepción sobre el nivel de discriminación y prejuicio presente en la sociedad, el nivel de sociabilidad de los mayores, ni tampoco en cuanto a la percepción sobre los problemas asociados a la vejez.

Asimismo, indicar que tras la participación de los seniors en el programa se ha detectado una mejora en su percepción sobre la capacidad de los propios mayores, así como a sus expectativas ante la vejez. Un aspecto que podría haber afectado es el hecho de que la participación en el programa supone tomar una actitud activa y un compromiso. Del mismo modo, el contacto con otras personas mayores de diferentes edades podría haber influenciado positivamente; los seniors participantes tenían entre 55 y 84 años, el contacto con otras personas de mayor edad, que tenían gran energía y nivel de actividad, podría haber influido positivamente a los seniors más jóvenes en cuanto a sus propias expectativas hacia la vejez.

A la luz de los resultados obtenidos, algunas cuestiones a las que cabría dar respuesta en futuras investigaciones incluyen: ¿qué opinan los niños y los sénior sobre su propio proceso? ¿perciben ellos mismos cambios en sus actitudes?, ¿se detectan cambios en sus discursos tras la participación en el programa? En este sentido, una futura línea de trabajo podría ir dirigida a analizar las creencias implícitas de los participantes y a su asignación de significados a través de técnicas cualitativas de recogida de datos. Disponer de una medida sobre las actitudes implícitas mitigaría la deseabilidad social en las respuestas.

Con todo, se debe considerar la necesidad de seguir investigando y evaluando los programas que se implementan en los centros escolares para poder disponer de programas validados para diferentes contextos y en diferentes cursos de Educación Primaria, con el fin de implantar programas sostenibles que promuevan el contacto y la relación entre generaciones, que se antoja beneficiosa para todos los participantes. 


\section{Agradecimientos}

Estudio realizado en el marco del Proyecto "Sharing Childhood-Sachi2" con referencia 2016-1-ES01KA204-024999, financiado por el Programa Erasmus+ del Servicio Español para la Internacionalización de la Educación (SEPIE).

\section{Referencias}

Babcock, R. L., MaloneBeach, E. E., Hannighofer, J. y Woodworth-Hou, B. (2016). Development of a children's IAT to measure bias against the elderly. Journal of Intergenerational Relationships, 14(3), 167-178. https://doi.org/10.1080/15350770.2016.1195245

Babcock, R. L., MaloneBeach, E. E. y Salomon, H. (2018). A quantitative and qualitative evaluation of the impact of an intergenerational program on children's biases toward older adults. Journal of Intergenerational Relationships, 16(2), 123-138. https://doi.org/10.1080/15350770.2018.1404423

Blasco-Serrano, A. C., Dieste, B. y Coma, T. (2019). Actitudes en centros educativos respecto a la educación para la ciudadanía global. REICE. Revista Iberoamericana sobre Calidad, Eficacia y Cambio en Educación, 17(3), 79-98. https://doi.org/10.15366/reice2019.17.3.005

Chung, S. y Kim, J. (2020). The effects of intergenerational program on solidarity and perception to other generations in Korea. Journal of Social Service Research, 47(2), 219-231. https://doi.org/10.1080/01488376.2020.1744501

Cohen-Mansfield, J. y Jensen, B. (2017). Intergenerational programs in schools: Prevalence and perceptions of impact. Journal of Applied Gerontology, 36(3), 254-276. https://doi.org/10.1177/0733464815570663

Connolly, P. (2006). Quantitative data analysis in education. Routledge. https://doi.org/10.4324/9780203946985

Cummings, S., Williams, M. y Ellis, R. (2008). Impact of an intergenerational program on 4th graders' attitudes toward elders and school behaviors. Journal of Human Behavior in the Social Environment, 6(3), 91-107. https://doi.org/10.1300/J137v06n03_06

Doiron, R. y Lees, J. (2009). It takes a village to raise a reader: Reflections on an intergenerational literacy program. The School Community Journal, 19(1), 137-154.

Dunham, C. C. y Casadonte, D. (2009). Children's attitudes and classroom interaction in an intergenerational education program. Educational Gerontology, 35(5), 453-464. https://doi.org/10.1080/03601270802605473

Education and Training. (2020). EU policy in the field of adult learning. https://ec.europa.eu/education/policies/eu-policy-in-the-field-of-adult-learning_en

Fujiwara, Y., Sakuma, N., Ohba, H., Nishi, M., Lee, S., Watanabe, N., Kousa, Y., Yodhida, H., Fukaya, T., Yajima, S., Amano, H., Kureta, Y., Ishii, K. y Uchida, H. (2009). Effects of an intergenerational health promotion program for older adults in Japan.Journal of Intergenerational Relationships, 7(1), 17-39. https://doi.org/10.1080/15350770802628901

Gamilel, T. y Gabay, N. (2014). Knowledge exchange, social interactions, and empowerment in an intergenerational technology program at school. Educational Gerontology, 40(8), 597-617. https://doi.org/10.1080/03601277.2013.863097

George, D., Whitehouse, C. y Whitehouse, P. (2011). A model of intergenerativity: How the intergenerational school is bringing the generations together to foster collective wisdom and community health. Journal of Intergenerational Relationships, 9(4), 389-404. https://doi.org/10.1080/15350770.2011.619922

Giraudeau, C. y Bailly, N. (2019). Intergenerational programs: What can school-age children and older people expect from them? A systematic review. European Journal of Ageing, 16(3), 363-376.

https://doi.org/10.1007/s10433-018-00497-4

Gualano, M. R., Voglino, G., Bert, F., Thomas, R., Camussi, E. y Siliquini, R. (2018). The impact of intergenerational programs on children and older adults: A review. International Psychogeriatrics, 30(4), 451-468.

https://doi.org/10.1017/S104161021700182X 
Guerra, M. D. (2014). La reducción de la estereotipia y el prejuicio sobre hombres y mujeres mayores en alumnado de educación secundaria: moduladores y líneas de actuación [Tesis doctoral, Universidad de Córdoba]. Archivo de la Universidad de Córdoba.

Gutiérrez Sánchez, M. y Hernández Torrano, D. (2013). Los beneficios de los programas intergeneracionales desde la perspectiva de los profesionales. Pedagogía Social. Revista Interuniversitaria, 21, 213-235.

Hatton-Yeo, A. (2015). A personal reflection on the definitions of intergenerational practice. Journal of Intergenerational Relationships, 13(4), 283-284. https://doi.org/10.1080/15350770.2015.1058319

Isaki, E.y Harmon, M.T. (2015). Children and adults reading interactively: The social benefits of an exploratoryintergenerational program. Communication Disorders Quarterly, 36(2), 90-101. https://doi.org/10.1177/1525740114529154

Kaplan, M. (2001). School-based intergenerational programs. UNESCO.

Kogan, L. R. y Schoenfeld-Tacher, R. M. (2018). Participation in an intergenerational service learning course and implicit biases. Educational Gerontology, 44(2), 90-98. https://doi.org/10.1080/03601277.2017.1413784

Lozano Martínez, J., Ballesta Pagán, F. J., Castillo Reche, I. S. y Cerezo Máiquez, M. C. (2018). El vínculo de la escuela con el territorio: Una experiencia de inclusión educativa. Profesorado. Revista de Currículum y Formación del Profesorado, 22(2), 207-226. https://doi.org/10.30827/profesorado.v22i2.7720

Lynott, P. y Merola, P. (2007). Improving the attitudes of 4 graders toward older people through a multidimensional intergenerational program. Educational Gerontology, 33, 63-74. https://doi.org/10.1080/03601270600864041

Montoro, J. (1998). Actitudes hacia las personas mayores y discriminación basada en la edad. Revista Multidisciplinar de Gerontología, 8(1), 21-30.

Moreno Abellán, P., Martínez de Miguel López, S. y Escarbajal de Haro, A. (2018). El impacto educativo de los programas intergeneracionales: un estudio desde la escuela y las diferentes instituciones sociales implicadas. Revista Iberoamericana de Educación, 77(2), 31-54. https://doi.org/10.35362/rie7723158

Murayama, Y., Ohba, H., Yasunaga, M., Nonaka, K., Takeuchi, R., Nishi, M., Sakuma, N., Uchida, H., Shinkai, S. y Fujiwara, Y. (2015). The effect of intergenerational programs on the mental health of elderly adults. Aging and Mental Health, 19(4), 306-314. https://doi.org/10.1080/13607863.2014.933309

Newman, S. y Hatton-Yeo, A. (2008). Intergenerational learning and the contributions of older people. Aging Horizon, 8, 3139.

Newman, S., Ward, C., Smith, T., Wilson, J. y Mc Crea, J. (1997). Intergenerational programs: Past, present, and future. Taylor and Francis.

Orozco, I. y Moriña, A. (2020). Estrategias metodológicas que promueven la inclusión en educación infantil, primaria y secundaria. Revista Internacional de Educación para la Justicia Social, 9(1), 81-98.

https://doi.org/10.15366/riejs2020.9.1.004

Orte, C., Vives, M., Amer, J., Ballester, L., Pascual, B., Gomila, M. A. y Pozo, R. (2018). Sharing intergenerational relationships in educational contexts: The experience of an international program in three countries (Spain, Poland and Turkey). Journal of Intergenerational Relationships, 16(2), 86-103. https://doi.org/10.1080/15350770.2018.1404414

Osborne, P.y Hall, S. (2008) The impact of short-term quality intergenerational contact on children's attitudes toward older adults. Journal of Intergenerational Relationships, 5(4), 59-76. https://doi.org/10.1300/J194v05n04_05

Pinazo, S. y Kaplan, M. (2007). Los beneficios de los programas intergeneracionales. En M. Sánchez Martínez (Coord.), Programas intergeneracionales. Hacia una sociedad para todas las edades. Fundación "la Caixa".

Pinazo, S. y Montoro, J. (2008). An intergenerational program "Nau Gran” at the University of Valencia (Spain). Journal of Intergenerational Relationships, 1(2), 103-104. https://doi.org/10.1300/J194v01n02_10

Sánchez, M., Sáez, J., Díaz, P. y Campillo, M. (2018). Intergenerational education in Spanish schools: Making the policy case. Journal of Intergenerational Relationships, 16, 166-183. https://doi.org/10.1080/15350770.2018.1404859

Thompson, E. H. y Weaver, A. J. (2016). Making connections: The legacy of an intergenerational program. Gerontologist, 56, 909-918. https://doi.org/10.1093/geront/gnv064 
Veelken, L. (2001). Alemania. En A. Hatton-Yeo y T. Ohsako (Eds.), Programas intergeneracionales: política pública e implicaciones de la investigación. Una perspectiva internacional (pp. 25-30). UNESCO.

Vives, M., Ballester, L. y Valero, M. (2016). Efectos de los proyectos intergeneracionales en las escuelas. El ejemplo de Sharing Childhood. En C. Orte y M. Vives (Eds.), Compartir la infancia. Proyectos intergeneracionales en las escuelas (pp. 95-106). Octaedro.

Whiteland, S. R. (2016). Exploring aging attitudes through a puppet making research study. International Journal of Education \& the Arts, 17(3), 1-29.

Whitehouse, P. J. (2013). The challenges of cognitive aging: Integrating approaches from neuroscience to intergenerational relationships. Journal of Intergenerational Relationships, 11(2), 105-117.

https://doi.org/10.1080/15350770.2013.782740

Whitehouse, P. J., Bendezu, E., Fallcreek, S. y Whitehouse, C. (2000). Intergenerational community schools: A new practice for a new time. Educational Gerontology, 26(8), 761-770. https://doi.org/10.1080/036012700300001412

Whitehouse, C., Whitehouse, P. J. y Sánchez, M. (2016). Intergenerational reading rooms: Lessons learned from the intergenerational schools. En M. Kaplan, L. L. Thang, M. Sánchez y J. Hoffman (Eds.), Intergenerational contact zones. A compendium of applications. Penn State Extension.

\section{Breve CV de los/as autores/as}

\section{Carmen Orte}

Catedrática de Universidad del Departamento de Pedagogía y Didácticas Específicas. Licenciada en Psicología y Doctora en Ciencias de la Educación por la Universitat de les Illes Balears. Directora del Máster de Intervención Socioeducativa en Infancia, Adolescencia y Familia de la UIB. Creadora y directora del Anuario del Envejecimiento de las Islas Baleares. Directora del Laboratorio de Familia (LIFAC) de la UIB. Investigadora del Principal del Grupo de Investigación GIFES (Grup d'Investigació i Formació Educativa i Social) (http://gifes.uib.eu) de la UIB. Trabaja en los ámbitos de la prevención familiar en programas educativos basados en evidencia, la gerontología educativa y social y la pedagogía social.

Email: carmen.orte@uib.es

ORCID ID: https://orcid.org/0000-0002-4695-441

\section{Margarita Vives}

Graduada en Magisterio, Licenciada en Psicopedagogía y Doctora con Mención Europea en Ciencias de la Educación por la Universitat de les Illes Balears. Contratada doctora de Departamento de Pedagogía y Didácticas Específicas. Entre sus líneas de investigación principal están la calidad de vida, el apoyo social a las personas mayores, el aprendizaje en el largo de toda la vida, la inadaptación infantil y juvenil y gestión de conflictos y la mediación. Uno de los ejes de su trayectoria profesional ha sido la vinculación con el Grupo de Investigación y Formación Educativa y Social (GIFES). Email: marga.vives@uib.es

ORCID ID: https://orcid.org/0000-0001-6059-0717

\section{Joan Amer}

Profesor Titular de Universidad del Departament de Pedagogía y Didácticas Específicas. Licenciado y Doctor en Sociología por la Universitat Autònoma de Barcelona. Director del Anuario de la Educación de las Islas Baleares (Colonya-Caixa Pollença/UIB). Subdirector del Máster en Intervención Socioeducativa en Infancia, Adolescencia y Familia de la UIB. Pertenece al Grupo de Investigación GIFES (Grup d'Investigació i Formació Educativa i Social) (http://gifes.uib.eu) de la UIB. Trabaja en los ámbitos de los programas educativos familiares basados en evidencia, la pedagogía social, la sociología de la familia y de la educación. Email: joan.amer@uib.cat

ORCID ID: https://orcid.org/0000-0001-7751-7110 


\section{Victoria Quesada}

Doctora en Educación (Ciencias Sociales y Jurídicas), con Mención Europea, por la Universidad de Cádiz. Licenciada en Pedagogía (UIB) y máster en Orientación y Evaluación SocioEducativas (UCA). De 2010 a 2017 trabajó como investigadora y docente en el área de Métodos de investigación y Diagnóstico en Educación de la Universidad de Cádiz. Miembro del grupo de investigación EVALfor (Evaluación en Contextos Formativos). Actualmente es Profesora Contratada Doctora del Departamento de Pedagogía y Didácticas Específicas de la Universitat de les Illes Balears, siendo también miembro de GIFES. Sus líneas de investigación se centran en la evaluación educativa participativa y la evaluación de programas socioeducativos. Email: victoria.quesada@uib.eu

ORCID ID: https://orcid.org/0000-0002-8881-2358 